


$$
\begin{aligned}
& 35 \\
& 4 \\
& 5
\end{aligned}
$$







\title{
Beitrag zur Kenntniss
}

der

\section{jüngsten Ammoneen}

\author{
Norddeutschlands
}

Dr. Clemens Schlüter.

\section{B on n,}

Verlag von A. Henry.

1867. 


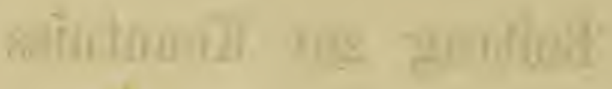

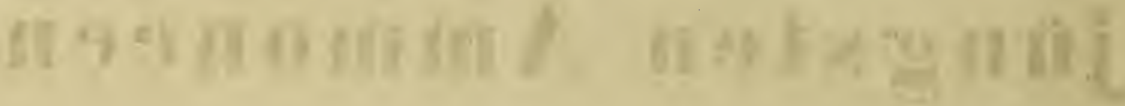

mowarestihirar

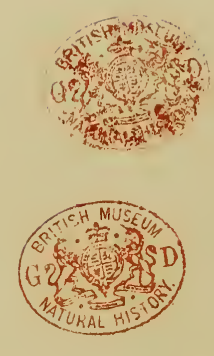

Bonn, Druck von Carl Georgi. 
Das Studium der mesozoischen Gebirgsschichten in Norddeutschland hatte seit den Fundamentalarbeiten vorzüglich von Goldfuss, Hoffmann und Römer längere Zeit lindurch fast geruht und namentlich im Vergleich mit Nachbarländern nur geringe Fortschritte aufzuweisen. In jüngerer Zeit ist auch in diesem Gebiete der vaterländischen Geologie ein neuer Aufschwung eingetreten. Mit ebenso grossem Eifer als Erfolge sind zunächst die jurassischen Gesteine ihren organischen Einschlüssen sowie ihren Lagerungsverhältnissen und ihrer Verbreitung nach studirt worden, wovon die Arbeiten der Herrn Brauns, Credner, F. Römer, Schlönbach und von Seebach beredte Beweise liefern. Die Ueberzeugung, dass das Parallelisiren ganzer Schichtengruppen nicht genüge. dass das scharfe Erkennen der Species (ihren zoologischen Eigenthümlichkeiten, wie ihrem geologischen Vorkommen nach) zur genaueren Schichteneintheilung und zum Wiedererkennen des an einem Orte durch bestimmte Arten characterisirten Niveaus in anderen Gegenden erforderlich sei. war maassgebend.

Dasselbe gilt für die Kreideperiode. Seitdem für die alten. weiten Glieder dieser Periode durch die Untersuchungen des Herrn von Strombeck eine präcisere Eintheilung geboten ist, sind auch die fossilen Reste derselben Gegenstand erneuter und schärferer Unter- 
suchung „zeworden. um sie noch weiter für die Geognosie nutzbar zu machen.

Für die bessere Kenntniss der Spongien hat bereits $A$. R ömer ${ }^{1}$ einen wichtigen Beitrag geliefert.

Die Foraminiferen haben in dem ausgezeichneten Kenner derselben $\mathrm{Aug}$. Reuss ${ }^{2}$ in Wien einen Bearbeiter gefunden.

Die Korallen wurden von W. Bölsche ${ }^{3}$ bearbeitet.

Die Brachiopoden sind von H. Credner ${ }^{4}$ und M. Schlönb a ch ${ }^{5}$ behandelt worden.

Ueber Crustaceen ${ }^{6}$ habe ich selbst einen Aufsatz nebst Nachtrag dazu geliefert.

Die Fische endlich sind durch W. von der Marck ${ }^{7}$ beschrieben worden.

1 Adolph Römer, Die Spongitarien des norddeutschen Kreidegebirges. Nit 19 Taf. $4^{0}$. (Palaeontographica.) Cassel 1864.

$2 \mathrm{Aug}$. Reus, Die Foraminiferen der westphälischen Kreideformation. Mit 13 Taf. $8^{\circ}$. (Sitzungsber. der math.-naturw. Classe der kais. Akad. der Wiss. Bd. XL, S. $147 \mathrm{ff}$.) Wien 1860.

A ug. Reuss, Die Foraminiferen des norddeutschen Hils und Gault. Mit 13 Taf. 89. (ebend. Bd. XLVI.) Wien 1862.

3 Will. Bölsche, Dic Korallen des norddeutsclien Jura- und Kreidegebirges. Mit 3 Taf. $8^{0}$. (Zeitschr. d. deutsch. geol. Gesellsch. Bd. XVIII. S. $439 \mathrm{ff}$. Berlin 1866.)

4 Herm. Credner, Die Brachiopoden der norddeutschen Hilsbildungen. Mit 2 Taf. (Zeitschr. d. deutsch. geol. Gesellsch. Bd. XVI. Berlin 1865.)

5 I. S chlönbą ch, Kritische Studien iiber Kreidebrachiopoden. Mit 3 Taf. (Palacontographica. Bd. XIII.) Cassel 1866.

M. Schlönbach, Ueber die Brachiopoden aus dem unteren Gault von Ahaus in Westphalen. (Zeitschr. d. deutsch. geol. Gesellsch. 1866. S. 365 ff.)

6 Cl. S chlüter, Die Makruren Dekapoden der Scnon- und Cenomanbildungen Westphalens. Mit 4 Taf. (Zeitschr. d. deutsch. geol. Gesellsch. 1862. S. 702 ff.)

7 W. von der Marck, Fossile Fische, Krebsc und Pflanzen aus dem Plattenkalke der jüngsten Kreide in Westphalen. Mit 4 Taf. (Palaeontograplica.) Cassel 1863.

W. v. d. Marck und Cl. Schliiter, Neue Fische und Krebse aus der Kreide Westphalens. (Palaeontographica.) Cassel 1867. 
Für die Characteristik und Altersbestimmung der einzelnen Schichten in der Kreideformation sind unter den fossilen Einschlüssen die Cephalopoden, wie allgemein anerkannt, von hervorstechender Wichtigkeit. Seit längerer Zeit habe ich dem Vorkommen derselben eine besondere Aufmerksamkeit gewidmet, so dass ich hoffe, durch die Mittheilung meiner in loco angestellten Beobachtungen, welche durch Benutzung der Sammlungen paläontologischer Gönner und Freunde noch ergänzt werden, einzelne Lücken in der bisherigen Kenntniss dieser Thierklasse auszufüllen.

Beiträge zur Kenntniss der Kreidecephalopoden werden mit dem vorliegenden Hefte eröffnet.

Die Hefte werden sich so rasch folgen, als es das Zeichnen und Lithographiren der Tafeln gestattet.

Was die Anordnung des zur Mittheilung kommenden Materials anbelangt. so erscheint es unthunlich, die Ammoniten familienweise zu gruppiren, da nicht das gesammte Material auf einmal zur Bearbeitung zu Gebote steht. Denn ist dies auch mit der eigenen Sammlung der Fall, so kōnnen die Exemplare fremder Sammlungen doch nur vor und nach benutzt werden. Für den Gebrauch des Buches möchte dieser Umstand sich als bequem erweisen, indem es möglich sein wird, die einzelnen Arten nach den Lokalitäten, und damit zugleich nach ihrem geognostischen Vorkommen zusammen zu stellen.

Von jeder Art ist mit der möglichsten Genauigkeit der Fundpunkt und die horizontale wie vertikale Verbreitung angegeben worden.

Für die einzelnen Schichten sind die gegenwärtig in Norddeutschland gebräuchlichen Benennungen angewandt.

1. Schichten mit Belemnitella mucronata.

2 . quadrata. 
Einleitung.

3. Schichten mit Epiaster brevis und Inoceramus Cuvieri.

4. $\quad$ M Micraster Leskei u. Spondylus spinosus, Scaphites Geinitzi.

5. $\quad$ Inoccramus Brongniarti und Ammonites Woolgari incl. Galeritcn-Pläner.

6. $\quad$ Inoceramus mytiloides (= labiatus) und Ammonites Cunningtoni.

i. $\pi$ Ammonitcs Rotomagensis und Discoidea cylindrica.

8.,$\quad$ Ammonites varians und Mantelli.

9. $\quad$ " Pecten asper (Tourtia oder Grünsand von Essen).

Es entsprechen die Schichten

1 und 2 der oberen Kreide oder dem Senon;

3, 4, 5, 6 dem obcren Pläner oder dem Turon;

7, 8, 9 dem unteren Pläner oder dem Cenoman. ${ }^{1}$

Es ist hier nicht der Ort, näher auf die Scheidung und die Reihenfolge der Schichten in der norddeutschen Kreideformation einzugehen. Es wird in den meisten Fällen bei näherer Angabe des Vorkommens genügen, den vorbenannten Schichten die Bezeichnung obere. mittlere, untere beizufügen.

Die mächtige Folge grauer und gelblicher Mergel im südlichen Westphalen bei Altenessen, Stoppenberg, Herne, Castrop schliesst sich als tiefstes Glied der Quadraten-Kreide an, und werden dieselben als unterstes Senon bezeichnet.

Die durch ihren Reichthum an fossilen Resten wohlbekannten Kreidemergel, welche sich von Lette in Westphalen, über Coesfeld, Holtwick und Legden erstrecken, aus denen F. Rö mer ${ }^{2}$ vorzugsweise

1 Ueber dic Verbreitung diescr Schichtcn im westphälischen Kreidebecken ist zu verglcichen: die Mittheilung des Verfasscrs in den Sitzungsberichten der niederrheinischen Gesellschaft fiir Natur- und Heilkunde (Verhandl. des naturhist. Ver. der Rheinlande und IVestplaten 1865) S. 125.

Ueber dic wichtigsten Fundpunkte in Hannover und Braunschweig ist von Strombeck (siche unten) und F. A. Römer „die ncucsten Fortschritte in der Mineralogic und Geognosie", Hannover 1865 S. 41 ff. zu vergleichen.

2 Die Krcidcbildungen von Westphalen. Verhandl. des naturhist. Vereins für Rheinland und Westphalen 1854. S. 140. 
Belemnitella mucronata citirt und welche auch Hosi us ${ }^{1}$ zu den Mucronaten-Schichten stellte, boten in all den zahlreichen Aufschlusspunkten kein Exemplar der Bel. mucronata, dagegen Bel. quadrata in grosser Zahl der Individuen. Sie bilden die jüngste Schichtenfolge der Quadraten-Kreide. Nicht minder reich an fossilen Resten sind übrigens die Kreidemergel mit Bel. mucronata, welche die obersten Schichten der Höhen zwischen Coesfeld und Darup bilden. Das Liegende derselben bildet nach den Aufschlüssen auf dem „Coesfelder Berge“ und „.Daruper Berge" ein als Baumaterial vielfach gewonnener Mergelsandstein, welcher verhältnissmässig nur sparsam fossile Reste umschliesst, unter diesen Bel. mucronata. Die im Innern der Baumberge bekannten Schichten, welche theils durch den Reichthum an fossilen Fischen, theils durch das häufige Vorkommen von Turrilites polyplocus ${ }^{2}$ characterisirt werden. dürften jünger sein.

Was die Terminologie der Ammoneenschalen anbetrifft, so dürfte es von allen Paläontologen für nothwendig erachtet werden, die bisherige Bezeichnungsweise zu verlassen, da beim lebenden Nautilus die convexe Seite dem Bauche, die concave dem Rücken des Thieres entspricht. Es wird also im Nachfolgenden die Siphonalseite als Bauch, die entgegengesetzte als Rücken bezeichnet werden, wie das bereits von Süss und Dittmar geschehen ist.

Um den lästigen Ballast der Citate nicht unnöthiger Weise zu vergrössern, wird kein Citat gegeben werden, welches sich einzig auf den Namen bezöge, nicht aber zugleich auch unsere Kenntniss der Art erweiterte.

1 Beiträge zur Geognosie Westphalens. ebend. 1860. S. 318.

2 A. Römer nennt freilich die Art auch aus den Hauptquadraten-Schichten von Dülmen. Diese Angabe ist irrthümlich. Tur. polyp. wurde in Westphalen niemals in anderen Schichten als den genannten gefunden und ist namentlich auch den oben genannten Mergeln von Coesfeld und Darup durchaus fremd. 
Lange bevor das Fundament für eine wissenschaftliche Betrachtung der fossilen Oroanismen gelegt war, hatten wie Versteinerungen überhaupt, so auch die Ammoneen im nördlichen Deutschland schon: Interesse erregt. Die aufgefundenen und für werthvoll gehaltenen Reste fanden mitunter solche Darstellungen, dass auch heute das Wiedererkennen keine Schwierigkeit bietet. So gedenken Nunning und Cohausen ${ }^{1}$ des riesigen Ammonites peramplus aus dem jüngsten Senon mit Belemnitella mucronata von Coesfeld als Cornu Ammonis; den als Cornu Bisontis bezeichneten Ammonites Rotomagensis fanden sie im cenomanen Pläner bei Oeding. Selbst die grossen Ancyloceren des erst neuerlich wieder entdeckten unteren Gault der Barler Berge bei Ahaus waren ihnen wohl bekannt. Wenn auch im weiteren Verlaufe der Zeit des Vorkommens von Ammoneen in jüngeren cretaceischen Sedimenten gedacht wird, so dürfen doch als wissenschaftlich begründet - nachdem durch Mantel und Sowerby eine Reihe Arten aus der Englischen und durch Brongniart aus der französischen Kreide bekannt gemacht waren - erst die Namen angesehen werden, welche F. W. Höning haus 1830 im Jahrbuche für Mineralogie, Geognosie und Petrefactenkunde von Leonhard und Bronn ${ }^{2}$ und H. von Dechen 1832 in seiner Bearbeitung des Handbuches der Geognosie von de la Beche veroffentlichten.

Höninghaus nennt folgende Arten:

1 Commercii literarii dissertationes epistologicae historico-physico-curiosae clarissimorum Westphaliae duumvirorum, Iodoci Hermanni Nunningii et Iohannis Henrici Cohausen de Glossopetris, Lapidibus Cordiformibus, Cornu Bisontis Petrefacto, Cornu Ammonis et Osse Femoris Elephantini etc. Cleomb. sec. Francofurti ad Moenum, anno MDCCXLVI.

2 Versuch einer geognostischen Eintheilung seiner Versteinerungssammlung von F. W. Höninghaus. Siebente Abtheilung. Grünsand, Kreide. II. Cephalopoden. S. 462 . 
Ammonites Buchii Hoen. Grünsand. Aachen. ${ }^{1}$

Lewesiensis So. Kreide. Essen.

Mantelli So. Grünsand. Bochum.

ornatus (Pollux). Grüinsand. Paderborn. ${ }^{2}$

rusticus So. Grünsand. Bochum. ${ }^{3}$

Selleguinus Bg. Kreide. Essen. ${ }^{4}$

varians So. Grünsand. Bochum.

Hamites intermedius So. Grünsand. Aachen.

rotundus Cuv.

Baculites Faujasii Lam. $\quad$ Bochum.

Sämmtliche hier aufgeführten Species werden auch von H. von Dechen (p. 432-346) mit Ausnahme des Amm. Buchii und Amm. ornatus citirt.

Die Zahl dieser Arten und Fundstellen hat eine wesentliche Erweiterung erfahren durch das Werk von Friedrich Adolph Römer, welches den Titel führt: Die Versteinerungen des norddeutschen Kreidegebirges. Mit 16 lithographirten Tafeln. Hannover 1841.

In diesem Werke werden aus der Kreide über dem Gault folgende Arten beschrieben und zum Theil auch abgebildet:

Ammonites peramplus Sow. Sarstedt, Alfeld, Goslar, Strehlen, Lemförde, Cocsfeld, Ilsenburg, Ahlten, Dülmen, Quedlinburg.

Ammonites Rotomagensis Brong. Osterfeld (irrthümlich), Rethen, Langelsheim.

Ammonites Mantelli Sow. Iburg, Sarstedt, Goslar, Quedlinburg, Liebenburg.

1 Die Art ist niemals beschrieben oder abgebildet worden.

2 Unverständliches Citat einer jurassischen Art. Weder Grünsand noch jurassische Schichten finden sich bei Paderborn, noch auch Formen von Ammoniten, welche mit Am. ornatus verwcchselt werden könnten.

3 Nach Vergleich der in Poppelsdorf liegenden Originale ergibt sich, dass dieselben zu Amm. Cunningtoni Sharpe gehören und aus dem Mytiloides-Pläner stammen.

4 Die Darstellung des Amm. Sclleguinus (Cuv. oss. foss. tab. VII, fig. 1) ist ungenügend für die Erkennung der Art. Die von Essen als solche bezeichneten Exemplare gehören zu Amm. planulatus Sow. = Amm. majorianus d'Orb. 
A mmonites bidorsatus Röm. Diilmen, Blankenburg. Ammonites falcatus Mant. Langelsheim, Waterlappe. Ammonites varians Sow. Sarstedt, Iburg, Goslar, Waterlappe. Ammonites coupei Brong. Sarstedt, Liebenburg. Scaphites a equalis Sow. Kromsberg bei Hannover. Scaphites obliquus Sow. Iburg.

Scaphites costatus Mut. Alfeld, Goslar, Liebenburg, Quedlinburg. Sc a phites inflatus Röm. Diilmen.

Sc a phites bidonosus Röm. Dülmen.

Scaphitcs compressus Röm. Ahlten. Scaphites plic atellus Röm. Lemförde. Scaphites pulcherrimus Röm. Lemförde, Vaëls. Scaphites ornatus Röm. Lemförde. Turrilites costatus Sow. Sarstedt, Rethen, Langelsheim.

Turrilites tuberculatus Sow. Langclsheim, Salzgitter, Alfeld.

Turrilites undulatus Sow. Peine, Rethen, Liebenburg.

Turrilites polyplocus Röm. Lemförde.

Hamites intermedius Sow. Aachen, Peine.

Hamites ellipticus Mant. Berne bei Hildesheim.

Hamites plicatil is Sow. Alfeld, Berne.

Hamites armatus Sow. Salzgitter.

Baculites Faujasii Lmk. Aachen, Lemförde.

Baculites anceps Lmk. Aachen, Blankenburg.

Baculites obliquatus Sow. Rethen, Langelsheim.

Baculites incurvatus Dujard. Quedlinburg.

Da das Werk von Römer die wichtigste (einheimische) Quelle für die Kenntniss der jüngsten Ammoneen des nördlichen Deutschland ist, wir also im weiteren Verlaufe unserer Arbeit gezwungen sind, stets darauf zurückzugreifen, so ist es überflüssig, schon hier den genannten Arten critische Bemerkungen zuzufügen. Ebenso abstrahiren wir davon, die gelegentlichen Notizen, welche über unsere jüngeren Ammoneen in verschiedenen Aufsätzen zerstreut sich finden, hier zusammen zu lesen, da es nur zu nutzlosen Wiederholungen führen würde. indem doch an den erforderlichen Stellen Rechenschaft daruber gegeben werden muss. Wir begnügen uns also damit, hier diese kleineren Arbeiten zugleich mit der benutzten 
inländischen und ausländischen Litteratur in chronologischer Folge namhaft zu machen.

1812-1830 J. Sowerby: Mineral Conchology of Great Britain voll. I-VI. $8^{0}$. With 609 plates. London.

1822 Cuvier et Al. Brongniart: Description géologique des environs de Paris. $4^{0}$. Avec un Atlas de 17 pll. Paris.

1822 G. Mantell: The fossils of the South Downs, or Illustrations of the geology of Sussex, $4^{0}$. W. 42 pll. London.

1827 Nilsson: Petrefacta Suecana formationis cretaceae. c. X tab. Londini Gothorum 1827.

1834 S. G. Morton: Synopsis of the Organic Remains of the Cretaceous group of the United States. Illustr. by 19 pl. $8^{\circ}$. Philadelphia.

1835 F. Dujardin: Mémoire sur les couches du Sol en Touraine, et description des coquilles de la Craie et des Faluns. Av. XX pl. (Mém. de la société géologique de France. Tome deuxième. Prem. part. p. 211-311.)

1840 Alc. d'Orbigny: Paléontologic Française. Description zoologique et geologique de tous les animaux mollusques et rayonnés fossiles de France. Terrains crétacées. Tom. I. Cephalopoda. $8^{0}$. Av. 148 pl. Paris.

1841 Friedr. Ad. Römcr: Die Versteinerungen des norddeutschen Kreidegebirges. Mit 16 Taf. $4^{0}$. Hannover.

1842 Fr. v. Hagenow: Monographie der Rügenschen Kreideversteinerungen. III. Abtheilung: Mollusken. (Neues Jahrbuch für Mineralogie etc. von Leonhard und Bronn. Jahrgang 1842. S. 528-575. Mit Taf. IX.)

1845 E. Forbes; Report on the fossil Invertebrata from Southern India, collected by Mr. Kaye and Mr. Cunliffe. p. 97-174. Tab. VII-XIX. ('Transaetions of the Geological Society of London. Scc. Ser. Vol. VII.) London 1845 .

1845-1846 Aug. Reuss: Die Versteinerungen der böhmischen Kreideformation. Mit 51 Taf. $4^{0}$. Stuttgart.

1846-1849 Fr. Quenstedt: Petrefactenkunde Deutschlands. Der ersten Abtheilung erster Band. Cephalopoden. $8^{\circ}$. Mit 36 Taf. in Fol. Tübingen.

1849-1850 H. B. Gcinitz: Das Quadersandsteingebirge oder Kreidegebirge in Deutschland. Mit 12 Taf. $8^{\circ}$. Freiburg.

1850 Alc. d'Orbigny: Prodrome de Paléontologie stratigraphique universelle des animaux mollusques et rayonnés faisant suite au cours élémentaire de Paléontologie et de Geologie stratigraphiques. Deuxième volume. $8^{0}$. Paris.

1850 H. B. Geinitz: Charakteristik der Schichten und Petrefacten des sächsischböhmischen Krcidegebirges, sowic der Verstcinerungen von Kieslingswalde. Mit 31 Taf. Neue Ausgabe. Leipzig 1850. Die erste Ausgabe in einzelnen Heften, welche 1839, 1840, 1842 und 1843 erschienen. 
1850 Fried. Ewald: Ueber die Gränze zwischen Neocom und Gault. (Zeitsehr. d. deutsch. geol. Gesellsch. Bd. II. S. 440-478.)

1850 Kner: Versteinernngen des Kreidemergels von Lemberg und seiner Umgebung. Mit 5 Taf. (Naturwissenschaftliche Abhandlungen gesammelt von Haidinger. III. Bd. 2. Abth. S. 1-42.)

$1850 \mathrm{Al}$. Alth: Geognostische Beschreibung der nächsten Umgebung von Lemberg. Mit 5 Taf. ebend. S. 171-284.

1851 Fred. Dixon: The geology and fossils of the Tertiary and Cretraceous Formation of Sussex. $4^{0}$. With $40 \mathrm{pl}$. London.

1852 Ferd. Römer: Die Kreidebildungen von Texas und ihre organischen Einschlüsse. Mit 11 Taf. $4^{0}$. Bonn.

1852 C. G. Giebel: Fauna der Vorwelt mit steter Berücksichtigung der lebenden Thicre. Dritter Band: Mollusken. Erste Abtheilung: Cephalopoden. $8^{0}$. Leipzig.

1852 Kner: Neue Beiträge zur Kenntniss der Kreideversteinerungen von OstGalizien. (Denkschriften der kaiserl. Akademie der Wissenschaften. Wien 1852. Bd. III. S. 293-335. Taf. 15-17.)

1853-1856 Dan. Sharpe: Description of the fossil remains of Molluska found in the Chalk of England. Part. I. Cephalopoda. 3 Abth. m. 27 Taf. (Mem. Paleont. Soc. London.)

1854 James Hall und Neck: Description of new Species of Fossils, from the Cretaceous Formations of Nebraska (Memoirs of the American Academy of Arts and Sciences. Cambridge a. Boston. Vol. V. P. 2. p: 379-411.)

1855 Will. Baily: Description of some Cretaceous Fossils from South Africa. (The quat. Journ. of the gcol. Soc. London. vol. XI. p. 454-465. With pl. XI-XIII.)

1856 E. Hebert: Foss. de la craie de Meudon. (Mém. de la soc. géol. de France. 2. sér. tom. V.)

1857 C. Giebel: Paläontologische Untersuchungen (Zeitschrift für die gesammten Naturwissensehaften von Giebel u. Heintz. Bd. X. S. 302-327.)

1857-1863 W. A. Ooster: Catalogue de céphalopodes fossiles des Alpes Suisses. Avec la description et les figures des espèces remarquables. Part. I-VI. av. $64 \mathrm{Pl} .4^{0}$. Genève.

1857 von Strombeck: Gliederung des Pläners im N. W. Deutsehland nächst dem Harze. (Jahrbuch von Leonhard u. Bronn. S. 785 ff.)

$1858 \ldots$ F. Pietet et $G$. Campiche: Description des fossiles du terrain crétacé des environs de Sainte-Croix. Gèneve.

1858 F. von Hauer: Ueber die Cephalopoden der Gosauschichten. (Hauer, Beiträge zur Paläontologie I. 1. S. 7-14. Taf. I-III.)

1859 von Strombeck: Beitrag zur Kenntniss des Pläners über der westphä- 
lischen Steinkohlenformation. (Zeitsehr. d. deutseh. geol. Gesellseh. Bd. XI. S. $27-78$.

$1861 \mathrm{~J}$. Binkhorst van den Binkhorst: Monographie des Gastéropodes et des Céphalopodes de la eraie supérieure du Limburg. a. 19 pl. $4^{0}$. Bruxelles et Naestrieht.

1862 F. von Hauer: Ueber die Petrefacten der Kreideformation des Bakonyer Waldes. Mit 3 Taf. (Sitzungsberiehte der kais. Akad. d. Wissensehaften in Wien. Bd. XLIV. S. 632-659.)

1863 Dreseher: Ueber die Kreidebildungen der Gegend von Löwenberg. (Zeitschr. d. deutsch. geol. Gesellsch. Bd. XV. S. 291.)

1863 von Strombeck: Ueber die Kreide am Zeltberge bei Liineburg. (Zeitsehr. d. deutseh. geol. Gesellseh. Bd. XV. S. 97.)

1864-1866 F. Stoliezka: The Fossil Cephalopoda of the Cretaeeous Roeks of Southern India. (Memoirs of the Geological Survey of India. Palaeontologia Indica. III. 1-13. p. 41-216. tab. 26-94.)

1864 Geological Survey of California. Palaeontology. Vol. I. Seetion IV. Deseription of the Cretaceous Fossils by W. Gabb. p. 57-217. tab. 9-32.

1865 F. A. Römer: Die Quadraten Kreide des Sudmerberges bei Goslar. ( $\mathrm{Pa}$ laeontographica. tom. XIII. p. 193-199. tab. 32.)

1865 Suess: Ueber Ammoniten. Sitzungsberichte der kais. Akad. d. Wissensch. Math.-naturwiss. Klasse LII. Erste Abth. 6. S. 71-90 erste Abth.

$1865 \mathrm{H}$. Seely: On Ammonites from the Cambridge Greensand. (Annales and Magazin of Natural History. Third ser. Vol. XVI. p. 225-247. tab. X. XI.)

1866 C. Schlüter: Die Schiehten des teutoburger Waldes bei Altenbeken. (Zeitschr. d. deutsch. geol. Gesellseh. Bd. XVIII. S. $35-76$. )

$1866 \mathrm{H}$. Sehlönbach: Ueber die Parallelen zwischen dem oberen Pläner Norddeutschlands und den gleiehalterigen Bildungen im Seinebecken. (Neues Jahrb. f. Mineral. ete. III. Heft.)

1866 v. Hauer: Neue Cephalopoden aus den Gosaugebilden der Alpen. Mit 2 Tafeln. Sitzungsberichte der kais. Akad. d. Wissensch. Math.-naturw. Klasse. LIII. Bd. Erste Abth. 3. p. 300-311. Mit 2 Tafeln. 



\section{AMMONITES Brug.}

AMMONITEN AUS SENON-SCHICHTEN. 


\title{
Ammonites Coesfeldiensis $\mathrm{n}$. sp.
}

\author{
Tab. I. Fig. 1-5.
}

Die Grösse dieses Ammoniten beträgt durchschnittlich 75 Millimeter. Die zwei oder drei flachen Umgänge des Gehäuses bilden einen so engen Nabel, dass nur etwa der dritte Theil ibrer Breite darin siehtbar ist. Die meisten Exemplare sind comprimirt; bei diesen ist die steile Nabelfläche verschwindend klein und geht mit sehwacher Biegung in die Seitenfläche über. Bei anderen Exemplaren, welche so von der umgebenden Gebirgsmasse eingeschlossen wurden, dass sie mit der Mündung auf der Schichtungsfläche liegen (Fig. 4) steht die ebene Nabelfläche senkrecht auf der Naht und biegt in einer rechtwinkeligen Kante zur Seitenfläche um. Die Seiten sind gewöhnlich flach, und nur das äussere Viertel oder Fünftel gegen die Bauchkante geneigt, oder schwach gewölbt, so dass die Schalcnöffnung ein längliches Oval bildet. Wie weit an dieser Form Verdrückung mitwirkt, ist nicht mit Sicherheit zu ergründen, da die quer im (iestein liegenden Exemplare auch wohl in Folge des Druckes bauchiger sind. (Siehe d. angez. Fig.) Die gegen die Bauchseite verschmälerte Mündung ist stets höher als breit. In der Jugend etwa ein 1) rittel (fig. 3), bei den grösseren Stücken mehr als die Hälfte. An der Nabelkante des letzten Imganges, zum Theil auf der Nabelfläche entspringend, liegen etwa 16 undeutliche Knoten, von denen ein, zwei, auch drei Rippen ausgchen und unter denen eine schärfer hervortritt als die übrigen. Diese Rippen sind in der Nabelgegend und auf dem unteren Theile der Seiten gering, im obersten Theile der Seiten gegen die Baurhfllichlie hin stark nach vorn gezogen. Indem hin und wieder 
noch einzelne kürzere Rippen sich einschieben, bleibt der Raum zwischen denselben ziemlich derselbe, kommt gewöhnlich der Breite einer Rippe gleich und ist nur im höheren Alter um ein Gcringes grösser. Bei Exemplaren von $75 \mathrm{~mm}$. Durchmesser zählt man 66 Rippen. Dort wo die Seiten sich mehr dem Bauche zuneigen, und wo zugleich die Rippen stärker nach vorn biegen, tragen dieselben einen kleinen runden Knoten. In der letzten Hälfte der äusseren Windung verschwinden dieselben. Ausser diesem Schmuck ist noch jede Kante des Bauches mit weit hervortretenden Zähnen besetzt, an deren Basis stets 2 oder 3 Rippen enden. Diese Zähne halten nicht bis zum vollendeten Wachsthum der Schale aus, sie werden auf dem letztem Drittel der äusseren Windung kleiner und bilden dann nur noch schwache runde Knötchen, und selbst diese verschwinden zuletzt auch wohl gänzlich $(4 \mathrm{c})$. Der Bauch ist flach oder bildet eine seichte Rinne, welche gewöhnlich glatt ist. Bei jüngeren Exemplaren zieht sich selten eine Andeutung der Rippen über dieselben hin: in höherem Alter ist dieses jedoch regelmässig der Fall (Fig. 1c u. 4c).

Von dieser Normalform finden sich bei kleineren und mittleren Exemplaren mehr oder minder erhebliche Abweichungen. Einige derselben sind unter Fig. 2, 3 und 5 abgebildet worden. Die Rippen sind hier weniger zahlreich, einfacher, in ihrem Verlaufe mehr geradlinig. Zuweilen ist die Verbindung der inneren und äusseren Knotenreihe so, dass man an gewisse Formen des Ammonites varians gemahnt wird (Fig. 3). Bei denjenigen Stücken, welche eine geringe Zahl von Rippen tragen, sind diese stärker und weiter abstehend als gewöhnlich, zugleich einfach radial, nicht oder nur schwach gebogen und nur selten durch Einschiebung oder Theilung vermehrt. 'Uebrigens' sind alle diese, nur selten auftretenden Formen durch Uebergänge mit der Normalform verbunden, wie dies schon das unter Fig. 5 abgebildete Exemplar anzeigt. Dahin sind auch noch die Stücke zu rechnen, welche weder Knoten, noch Zähne, die vielleicht beim Versteinerungsprocesse verloren gingen, tragen. Dergleichen Formen erinnern dann an Ammonites Lafresnayanus, den d'Orbigni aus dem Senon von Freville (Manche) abbildet (Terr. crét. tab. 97, fig. 3, 4.)

Die Lobenlinie ist an keincm Exemplar sichtbar. Der Untersuchung lagen 28 Exemplare zu Grunde. 

Zähne:

Die Ma asse eines grösseren Exemplars ohne die vorspringenden

\begin{tabular}{|c|c|c|c|c|c|c|c|c|c|}
\hline Durchn & messer der Scha & & . & . & & & & & \\
\hline Weite & des Nabels & & & . & & & . & & \\
\hline Höhe & des letzten Umg & ganges i & in $\operatorname{der} W$ & Tindun & & & & & \\
\hline ” & $n$ & " & von der & Naht & bis & zum & & auch & 38 \\
\hline & $\rrbracket$ vorletzten & & $\Rightarrow \quad n$ & " & " & $\pi$ & & 》 & 15 \\
\hline Involut & ter Theil des vo & rletzten & n Umgan & & & & & & 12 \\
\hline Dicke & des letzten Umg & ganges. & .. & & & & & & 15 \\
\hline$\pi$ & $\eta$ vorletzten & $\eta$ & . & . & . & & & & 6,5 \\
\hline
\end{tabular}

Geologisches Vorkommen. Die Art gehört der senonen Kreide mit Belemnitella mucronata an. Ich kenne sie bis jetzt nur aus der Gegend von Coesfeld. Dort gehört sie, in den nordöstlich und südöstlich von der Stadt verbreiteten jüngsten Kalkmergeln zu den häufigeren Vorkommnissen. Da der dortige zur Agricultur verwendete Mergel sehr leicht der Verwitterung erliegt und zerfällt, so gelingt es nur unter günstigen Umständen, wie oft man auch Spuren entdecken mag, Exemplare einzusammeln. In dem festeren, das Liegende jener Mergel bildenden Mergelsandstein habe ich die Art niemals beobachtet, ebenso wenig in den petrographisch sehr ähnlichen Mergeln mit Belemn. quadrata, welche von Legden und Holtwick herstreichend in der Nähe der Stadt ebenfalls eine schmale Zone bilden. Vorzügliche Fundstellen sind der Suekerhook und die Bauerschaft Harle. Auch in der Nähe von Darup habe ich die Art gesehen.

Alle Originale in meiner Sammlung.

\section{Erklärung der Abbildungen.}

Tafel I.

Fig. 1 Normales Exemplar, $1 \mathrm{a}$ von der Seite, $1 \mathrm{~b}$ gegen die Mündung, $1 \mathrm{e}$ gegen den Baueh gesehen.

Fig. 2 Varietät mit einfachen krättigen Rippen, $2 \mathrm{a}$ gegen die Seite, $2 \mathrm{~b}$ gegen die Mlïndung gesehen.

Fig. 3 a, b Junges Exemplar, gegen die Seite und gegen die Mündung gesehen.

Fig. 4 a, b Auf der Miundung liegendes, comprimirtes, sonst normal ausgebildetes Exemplar, gegen die Seite und gegen den Baueh gessehen.

Fig. 5 Varietät, gegen die Seite gesehen.

Sämmtliche Stiieke in natürlicher Grösse dargestellt. 


\section{Ammonites costulosus sp. n.}

Taf. II Fig. 1-4.

Die Schale mit einer durchschnittlichen Grösse von $95 \mathrm{Mm}$. ist scheibenförmig, involut, zusammengedrückt mit flachen oder wenig gewölbten Seiten, scharf begränztem schmalem Bauch, engem Nabel und zahlreichen gebogenen Rippen versehen. Die Rippen entspringen in Knoten an der Nabelkante und vermehren sich durch Einsetzen. Sie beginnen mit einer starken Richtung nach vorn, die sie etwa bis zur Mitte der Seiten innehalten, dann biegen sie sich schwach sichelförmig wenig rückwärts, um sich in der Nähe des Bauches wieder nach vorn zu wenden. Auf der abgeplatteten Bauchfläche selbst sind sie kaum sichtbar angedeutet, dagegen bilden sie an den scharfen, vom Bauch und den Seitenflächen gebildeten Kanten mehr oder minder deutliche, verlängerte zahnartige Vorsprünge. An einem Exemplar mittlerer Grösse zählt man etwa 46 Rippen auf dem letzten Umgange. Ausser diesen Rippen ist die Uberfläche noch mit feinen Reifen versehen, welche mit den Rippen parallel laufen, und dicht gedrängt, gleichmässig Seiten und Bauch, sowohl die Zwischenräume der Rippen, wie diese selbst bedecken. Bei einem Exemplar von $65 \mathrm{Mm}$. Durchmesser zählt man zwischen zwei Rippen 7 solcher Reifen.

Bei manchen Exemplaren sind die Rippen ${ }^{1}$ nur ausserordentlich schwach angedeutet oder sogar gar nicht vorhanden, wenn auch die Reifen deutlich sichtbar sind. So bei einem unter Figur 3 dargestellten Stücke. Muthmasslich sind dieselben erst beim Versteinerungsprocesse verloren gegangen, da sich Exemplare finden, denen auch die Reifen fehlen (Fig. 4). Von diesen Stücken, wie ein solches unter Fig. 4 abgebildet ist, dürfte diese Annahme unzweifelhaft und nicht etwa dem Jugendzustande zuzuschreiben sein, da man an der inneren Windung anderer Exemplare bei gleicher Grösse die Reifen wahrnimmt.

1 Die Figur 2 a ist nicht besonders gelungen, da die Rippen hier leicht für Vertiefungen der Oberfläche angesehen werden könnten. Ausserdem ist der involute Theil des vorletzten Umganges zu gering angegeben; siehe die Maasse. 
Das Vorkommen im Kalke von Ahlen (Fig. 1) zeigt flach gewölbte Seiten, wclche gegen die Bauchkante hin sanft zugerundet sind. Bei den Exemplaren aus den Mergeln von Cocsfeld (Fig. 2-4) bemerkt man dies nicht. Die flachen Seiten neigen sich hier unmerklich den Bauchkanten. Wie weit hier eine primäre oder sekundäre Erscheinung vorliegt, ist nicht $\mathrm{zu}$ ermitteln.

Masse:

Durchmesser der Schale

Weite des Nabels.

I.

II.

Höhe des letzten Umganges in der Windungsebene 27

$65 \mathrm{Mm}$. $132 \mathrm{Mm}$.

$12 n 30$ "

$", \quad$ vorletzten

Bauche
von der Naht bis zum
Bauche

Involuter Theil des vorletzten Umganges. .

Grösste Dicke des letzten Umganges . .

" $\quad$ vorletzten Umganges . . 3,5 ,

Eine äusserlich verwandte Form bietet: Ammonites Vibrayeanus d'Orb. terr. crét. pl. 96, fig. $1-3$ aus den Cénomanien von Vibrayes (Sarthe); doch ist die Ornamentik der Schale verschieden. Noch näher stehen einzelne Stücke dem Ammonites Orbignyanus Geinitz (Quader, tab.IV, fig. 1 und früher Charakteristik, Kieslingswalda, tab. I, fig. 8, und Stoliczka, fossil (ephalopoda tab. 48, fig. 2). Namentlich ist auch hier die Berippung verschieden. Die Rippen sind wenig zahlreich und statt vorwärts vielmehr rückwärts geneigt. Auch die Nähte der Kammerwände scheinen sehr verschieden zu sein. An einem der vorliegenden Exemplare glaube ich, freilich bei sehr ungünstiger Erhaltung, wahrzunehmen, dass ein grosser erster Lateral-Lobus, ein erheblich kleinerer zweiter und ein oder zwei sehr kleine Auxiliar-Loben vorhanden sind.

Geologische Verbreitung. Die Art gehört zu den weniger häufigen Ammoneen der Sichichten mit Belemnitella mucronata. Als Kalkversteinerung findet sie sich bei Ahlen in Westphalen. Ausserdem habe ich mehrere Exemplare in den Kreidemergeln von Coesfeld in Westphalen gefunden. Auch die diese Schichten unterteufenden Mergclsandsteine lieferten auch dem "C'oesfelder Berge" mehrere Stücke der Art.

Sämmtliche Originale in meiner Sammlung. 


\section{Erklärung der Abbildungen.}

Tafel II.

Fig. 1 a, b Grosses Exemplar mit flachgewölbten Seiten aus den festen Kalken von Ahlen in Westphalen von der Seite und gegen den Bauch gesehen.

Fig. 2 a, b Kleineres Exemplar mit abgeflachten Seiten aus den jüngsten Kalkmergeln von Coesfeld in Westphalen, von der Seite und gegen die Mündung gesehen.

Fig. 3 a, b Bruchstück eines Exemplars mittlerer Grösse ohne Rippen, nur mit den Haarreifen versehen, aus dem Mergelsandstein von Coesfeld.

Fig. 4 Junges Exemplar ohne Rippen und Reifen aus dem Kalkmergel von Coesfeld.

Alle Exemplare sind in natïrlicher Grösse abgebildet.

\section{Ammonites Haldemsis $s p$. $n$.}

Taf. III, Fig. 1 a. b.

Die drei Umgänge des $60 \mathrm{Mm}$. grossen Gehäuses sind zur Hälfte involut und haben eine niedrige, convexe Nabelfläche, über welcher die Seiten mit sehr geringer Wölbung bis zum Bauche sich ausdehnen. Die Höhe der Mündung übertrifft die Breite um ein sehr Erhebliches. Ueber der Nabelfläche des letzten Umganges - die inneren sind nicht gut conservirt - erheben sich 12-13 Rippen in fast gleichen Abständen, welche vollkommen gerade den Seiten leistenartig aufliegen und enden, ehe sie den Bauch erreichen. Auf der äusseren Hälfte der Windung werden diese Rippen von Knoten begränzt, die der Mündung ein wenig zugeneigt sind.

Die Nähte der Kammerwände sind zwar nicht mit Deutlichkeit erkennbar, doch deuten einzelne Spuren an dem Mergelkerne darauf hin, dass dieselben sehr zerschnitten waren. Die Wohnkammer ist an dem abgebildeten Stücke nicht erhalten.

Mass e :

Durchmesser der Schale . . . . . . $59 \mathrm{Mm}$.

Weite des Nabels . . . . . . . . . 22,

Höhe des letzten Umganges in der Windungsebene . . 18 ,

, ,, von der Naht bis zum Bauche 26 ,

, vorletzten, $, \pi, \pi, 11$,

Involuter Theil des vorletzten Umganges . . . 5 , 
Die Art steht den Ammonites Cunliffei Stoliczka (tab. 50, fig. 3, p. 97) aus der Ootatoor group Indiens nahe. Die Verschiedenheiten bestehen darin, dass bei der indischen Art der Bauch flacher und die Mündung niedriger ist, die Rippen etwas gekrümmt, nach vorn geneigt sind und auch schon in der Jugend mit einem Höcker enden.

Geologisches Torkommen. Ich kenne die Art bis jetzt nur aus der Hügelgruppe von Haldem und Lemförde (Hannover), deren Schichten durch das häufige Vorkommen von Belemnitella mucronata ihrem Alter nach charakterisirt sind.

Das abgebildete Exemplar befindet sich in der Sammlung des naturhistorischen Vereins der Rheinlande und Westphalens in Bonn.

\section{Erklärung der Abbildungen.}

Tafel III.

Fig. 1 Exemplar in natürlicher Grösse, 1 a von der Seite, 1 b gegen den Bauch und gegen die Mündung gesehen.

\section{Ammonites Proteus sp. n.}

$$
\begin{aligned}
& \text { 4. arritoedstatus fulut } \\
& + \text { tre } 4-y
\end{aligned}
$$

Taf. III, Fig. 2 a. b. c.

Alle Exemplare sind mehr oder minder verdrückt, so dass es schwer ist, von der ursprünglichen Gestalt ein vollkommen richtiges Bild zu erhalten. Doch scheint nach der Vergleichung sämmtlicher Stücke sich Folgendes mit ziemlicher Gewissheit zu ergeben.

Die Schale erreicht eine Grösse von circa $115 \mathrm{Mm}$. und besteht aus drei Umgängen, welche bei einigen Exemplaren etwas weniger, bei anderen etwas mehr als über die Hälfte involut erscheinen. Die convexe Nabelfläche ist steil und stösst in einer abgerundeten Kante gegen die Seitenfläche. Diese ist ziemlich flach, nur leicht gewölbt, wenig gegen den breiten, etwas abgeplatteten, kaum von den Seiten abgesetzten Bauch convergirend. Das ganze Gehäuse ist mit kräftigen Rippen versehen, welche in den verschiedenen Altersstadien eine verschiedene Entwicklung erleiden. In der Jugend, auf den inneren Windungen ziehen sich, an der Nabelkante beginnend, ziemlich fern stehende Rippen mit einer 
Neigung nach vorn über Seiten und Bauch. Auf letzterem mehrt sich durch Einschiebung einiger kürzeren Rippen die Zahl derselben. Einige Exemplare liegen vor, an denen je zwei dieser grösseren Rippen aus einem verlängerten, dornförmig ausgezogenen Knoten ausgehen. Beim Ansetzen der Wohnkammer gehen die Rippen nicht mehr über den Bauch, bilden aber an den Kanten dicke runde Höcker oder ohrartig erweiterte Vorsprünge. Ausserdem geht in demselben Stadium noch eine andere Teränderung mit den Rippen vor sich, indem sie zum Theil auf der Mitte der Seiten, oder etwas dem Nabel genähert, eine flache knotenartige Erweiterung oder Anschwellung erleiden. Hiermit ist noch eine fernere Erscheinung verbunden; der Theil der Rippen nämlich, welcher zwischen dieser Erweiterung und den äusseren Knoten liegt, verschmälert sich in der Regel und verschwindet zuweilen gänzlich, so dass dann an diesen Stellen die Schale, statt mit Rippen, mit Knoten geschmückt er'scheint. Diese Art der Ornamentik erinnert an ein indisches Vorkommen, an den freilich sonst ganz verschiedenen Ammonites Rotalinus Stoliczka, tab. 34, fig. 2. In diesen Stadium treten die Rippen am weitesten auseinander. - Im weiteren Wachsthum der Schale nähern sich die Rippen wieder und nehmen eine regelmässigere Gestalt an. Zunächst treten sie schwach auch auf dem Bauche zwischen den Knoten wieder hervor, und endlich im höchsten Alter laufen sie, ohne jegliche Spur von Knoten, als dicke, wulstartige Vorsprünge, an der Nabelfläche beginnend, ïber Seiten und Bauch hin. So gewinnt die Schale, je nach dem verschiedenen Standpunkte, von dem aus man sie betrachtet, ein ganz verschiedenes Ansehen.

Eine deutliche Lobenlinie wurde noch nicht beobachtet. Jedenfalls war dieselbe sehr complicirt, wio man an einigen undeutlichen Spuren erkennt. Als mir das erste Exemplar der Art zu Gesicht kam, hielt ich es für eine krankhafte, verkrüppelte Form. Nachdem ich aber selbst mehrere Exemplare gesammelt, andere in verschiedenen Sammlungen gefunden hatte, musste jene Meinung aufgegeben werden, da alle Stücke unter sich übereinstimmen, bei allen der so auffällige Wechsel in der Ornamentik erst mit Ansatz der grossen, 3/4 der Windung einnehmenden Wohnkammer sich findet.

Geologisches Vorkommen. Alle gesehenen und untersuchten Stücke stammen aus den Mukronaten-Mergeln von Haldem und Lem- 
Ammonites patagiosus.

förde (Hannover). Die zu vorstehender Beschreibung benutzten Exemplare befinden sich zum Theil im Museum des naturhistorisehen Vereins der Rheinlande und Westphalens in Bonn, zum Theil in meiner Sammlung.

\title{
Erklärung der Abbildungen.
}

Tafel III.

Fig. 2 nach einem etwas schräg gedrückten Exemplare restaurirtes Bild in natürlicher Grösse, 2 a gegen die Seite, 2 b gegen dic Seite und den Bauch, $2 \mathrm{e}$ gegen den Bauch gesehen, um den Wechsel in der Berippung und der Knotenbildung zu zeigen.

\section{Ammonites patagiosus $\mathrm{sp} . \mathrm{n}$.}

\author{
Taf. IV, Fig. 4,5 .
}

Das kleine Gehäuse besteht aus 2 bis 3 Umgängen, welche sich etwa zu zwei Drittel umschliessen und daher einen engen Nabel bilden. Umgänge ziemlich niedrig und breit. Der regelmässig gerundete Bauch verbindet sich ohne Spur einer Kante mit den Seiten. Die letzteren erheben sich mit steiler Nabelfläche über die Umgangsnaht und gehen mit gerundeter Kante in die convexen Seitenflächen über, so dass die Mündung, an Höhe und Breite nahezu gleich, eine abgerundet vierseitige, fast kreisförmige Gestalt annimmt, wobei von dem Ausschnitte des Rückens, der die vorige Windung aufnimmt, abgesehen wird. An der Umgangsnaht oder an der Nabelkante entspringen in ziemlich regelmässigen Abständen Furchen, welche fast geradlinig, mit schwacher Neigung nach vorn über Seiten und Bauch hinweglaufen. Diese Einschnürungen sind zugleich von einer Aufwulstung der Schale begleitet, wodurch der jedesmalige völlig ausgebildete Lippensaum ein halskragenartiges Ansehen gewinnt. An einzelnen Exemplaren sind stellenweise die Furchen doppelt vorhanden, und an den meisten Exemplaren beginnt die begleitende rippenartige Erhebung der Schale mit einem Knoten am Nabel. In der Jugend dürften nur diese Knoten vorhanden sein, und auch die Furchen garz fehlen. Im Uebrigen war die ganze Schale glatt. Es mag noch erwähnt werden, dass je nach der Erhaltungsart bald die Einschnü- 
rungen, bald die Rippen deutlicher sind. Die Zahl derselben schwankt, wie bei den verwandten Formen der unteren Kreide.

Die Nähte der Kammerwände sind an keinem Exemplar sichtbar.

Vielleicht steht der sehr ungenügend gekannte Ammonites Parisiensis Heb. ${ }^{1}$ aus der Kreide von Meudon nahe.

Maasse:

Durchmesser der Schale . . . . . . $51 \mathrm{Mm}$.

Weite des Nabels . . . . . . . . 17 ,

Höhe des letzten Umganges in der Windungsebene . . 15 ,

" $" \#$ von der Naht bis zum Bauche 21 ,

$\eta$ vorletzten $, \pi, \pi, \pi$,

Involuter Theil des vorletzten Umganges . . . 7 ,

Dicke des letzten Umganges . . . . . . 19 "

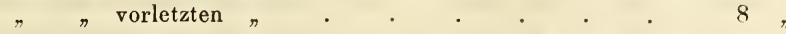

Die durchschnittliche Grösse des Gehäuses beträgt $50 \mathrm{Mm}$. Das Fragment eines grösseren Stückes zeigt an, dass die Art auch eine Grösse von $70 \mathrm{Mm}$. erreichen kann.

Zur Beschreibung lagen 11 mehr oder minder vollständige Exemplare vor.

Geologisches Vorkommen. Die Art gehört den Schichten mit Belemnitella mucronata an. Sie wurde bisher nur in den jüngsten kalkigen Mergeln der Gegend von Coesfeld (Westphalen) beobachtet.

Alle Originale in meiner Sammlung.

\section{Erklärung der Abbildungen.}

Tafel IV.

Fig. 4 und 5 stellen zwei vom Coesfelder Berge stammende Exemplare von der Seite und vom Bauche aus gesehen in natürlicher Grösse dar.

1 Mém. Soc. géol. Franc. II. Ser. tom. V, p. 369 tab. 29 fig. 2. 


\section{Ammonites Lettensis sp. $\mathrm{n}$.}

Taf. IV Fig. 3.

Steht der vorigen Irt durch (Grösse, Gestalt, Form und Zahl der Umgänge nahe, ist aber verschieden durch die stets fehlenden Einschnürungen, geringere Involubilität und deutliche Rippen. In der Jugend erscheinen die letzteren nur, als fern stehende verlängerte Knoten in der Gegend der gerundeten Nabelkante, die halbe Seitenhöhe kaum erreichend. Bei weiterem Wachsthum der Schale entwickeln sich die Rippen mehr und mehr, gehen über die Seiten und später auch über den Bauch weg. Zugleich schieben sich dann am Bauch einige Rippen zwischen, welche eine kurze Strecke auch noch auf den Seiten sichtbar sind. - Die Nähte der Kammerwände sind auch hier unbekannt.

Maas se:

Durchmesser der Schale . . . . . . . $47 \mathrm{Mm}$.

Höhe des letzten Umganges in der Windungsebene. . 16 n

,, von der Naht bis zum Bauche 19 ,

》 $\rightarrow$ vorletzten $, \pi, \pi$,,

Involuter Theil des vorletzten Umganges . . . . 3 ,

Dicke des letzten Umganges . . . . . . 21 ,

" vorletzten Umganges . . . . . . 11 ,

Verwandt ist die Art den jüngern Schalen von Amm. Portlocki (Sharpe p. 30, tab. XIII, fig. 3). Aber die Dicke der Windungen übertrifft hier bedeutend die Höhe; auch die Rippen sind zahlreicher und mehr gerade. - Auch Amm. Ganesa (Forb. Stol. p. 106. tab. 54, fig. 2) zeigt eine gewisse Aehnlichkeit. Bei dieser indischen Art entwickeln sich aber die Knoten niemals zu Rippen.

Zahl der untersuchten Stücke 4.

G e o logis ches Vorkommen. Die Art ist bis jetzt selten, ich habe nur wenige Exemplare in den obersten Mergelbänken mit Belemnitella quadrata zwischen Coesfeld und Lette in Westphalen sammeln können.

\section{Erklärung der Abbildungen.}

Tafel IV.

Fig. 3 a Exemplar in natürlicher Grösse gegen die Seite, $3 \mathrm{~b}$ gegen die Mündung und den Bauch gesehen. 


\section{Ammonites polyopsis.}

Taf. IV, Fig. 1, 2.

1833̃. Ammonites polyopsis Dujardin. Mém. soc. géol. France, tome II, p. 232, pl. VII, fig. 12 a, b, c.

1841. Ammonites bidorsatus, A.Römer, Verstein. d. norddeutsch. Kreidegeb. S. 88 , Taf. XIII, Fig. 8 .

,Scheibenförmig, genabelt, mit 2--3 schnell an Höhe zunehmenden, sehr involuten Windungen. An der Suturkante, welche steil abfällt, entspringen etwa 20 schwache Falten, welche sehr stark vorwärts gerichtet sind und auf dem ersten Viertheile der sehr schwach gewölbten Seite einen länglichen Höcker bilden; die Mehrzahl verschwindet dann und etwa acht erreichen fast den sehr schmalen Bauch, in dessen Nähe sie zu einem zweiten, von vorn nach hinten gerichteten Höcker anschwellen und dann aufhören; der Rücken trägt eine tiefe Längsfurche, welche von zwei scharfen Kanten begränzt ist." Dieser Beschreibung von Adolph Römer sind einige Bemerkungen zuzufügen. An den inneren Windungen habe ich weder Rippen noch Knoten beobachtet. Diese Ornamentik der Schale scheint erst ein Produkt des mittleren und späteren Alters zu sein. Die kurzen an der Nabelkante entspringenden Falten habe ich nur auf der Wohnkammer gcsehen. Die verlängerten Knoten in der Nähe des Bauches, welche sich der Bauchkante allmählig mehr und mehr nähern, kennc ich nur auf den letzten ${ }^{2} / 3$ der äusseren Windung. An einzelnen Exemplaren alterniren diese Knoten auf beiden Seiten. Dass von diesen Knoten Rippen über die Seiten weg zum Nabel laufen, habe ich niemals observirt. Dagegen zeigen sich wohl undeutliche Wellen auf den Seiten, wie unsere Abbildung sie angibt. Die Bildung des Bauches ist auch wechselnd. Die Längsfurche ist nicht immer wahrzunehmen. Zuweilen scheint es, als wenn dieselbe - wie Dujardin in der That angibt - von kleinen, auf den Kanten stehenden Knoten eingefasst wäre. Der Bauch ist auch keineswegs immer schmal, wie an den abgebildeten Exemplaren. Ich besitze ein Bruchstück, an dem die Breite $17 \mathrm{Mm}$. beträgt und erinnere mich, in der Sammlung des Herrn Dr. Ewald in Berlin Stücke gesehen zu haben, deren Bauch einen Zoll 
Breite besass. Wahrscheinlich ragte der Bauch stark an der Mündung vor, wie dies Anwachsstreifen vermuthen lassen, welche noch an einem Steinkerne sichtbar sind. Auch Dujardin gab schon ein solches Bild. Die Eigenthümlichkeiten der Lobatur sind aus der Abbildung derselben ersichtlich.

\section{Mass e :}

Durehmesser der Sehale. . . . . . . $124 \mathrm{Mm}$.

Weite des Nabels. . . . . . . . 25 n

Höhe des letzten Umganges in der Windungsebene . . 36 "

$n \pi n$ von der Naht bis zum Bauche 58 n

" vorletzten $" n, \quad$ ", 30 ,

Involuter Theil des vorletzten Umganges . . . 25 "

Grösste Dieke des letzten Umganges . . . . 27 n

" " vorletzten Umganges . . . . 11"

Geologisches Vorkommen. Die Art gehört den mittleren Schichten mit Bel. quadrata an, wo Dülmen in Westphalen den Hauptfundpunkt bildet. Auch Blankenburg am Harze bildet einen bekannten Fundpunkt. Nach Dujardin ebenfalls in der Kreide der Tourraine.

Die abgebildeten Exemplare in meiner Sammlung.

\section{Erklärung der Abbildungen.}

Tafel IV.

Fig. 1 Seitenansicht eines Exemplares mit zum Theil erhaltener Wohnkammer, 2a Ansieht eines grösseren Exemplares gegen den Bauch und gegen eine Kammerwand gesehen, $2 \mathrm{~b}$ die Nähte der Kammerwände des unter Fig. 2 a dargestellten Stüekes.

Alle Abbildungen in natürlieher Grösse.

\section{Ammonites tridorsatus n. sp.}

\section{'I'af. V Fig. 1.}

Die mässig grosse Schale besteht aus etwa fünf langsam an Höhe und Breite zunehmenden vierseitigen Umgängen, welche sich fast nur berühren, so dass ein sehr weiter Nabel offen bleibt. Die Seiten sind flach und fallen gegen den Nabel zu fast senkrecht ab. Querschnitt der inneren Windungen quadratisch, der äusseren oblong (NB. in der Abbildung 
etwas zu hoch angegeben), höher als breit. Die Seitenflächen sind bedeckt mit regelmässigen starken, nahezu radialen Rippen. Die Rippen sind alle einfach, an der Suturfläche bis zum ersten Knoten leicht gebogen, auf den Seiten gerade, 27 auf dem letzten, 25 auf dem vorletzten Lmgange, in regelmässigen Abständen stehend, der Zwischenraum zwischen zwei Rippen etwas grösser, als die Breite einer Rippe, jede zwei Knoten tragend. Die Knoten der inneren spiralen Reihe sind rund und stehen in einiger Entfernung von der Nabelkante; auf den inneren Windungen werden sie allmählig undeutlicher. In den Knoten der äusseren Spirale endigen die Rippen gegen den Bauch hin, wo sie stumpf abschneiden. Auf der Mittellinie des breiten, fast flachen Bauches erhebt sich ein scharfer, ununterbrochener, von zwei Furchen eingefasster Kiel. Jede Furche ist nach auswärts wiederum von einem Kiele begränzt. Die Nähte der Kammerwände sind unbekannt.

Masse:

Durchmesser der Schale . . . . . . $124 \mathrm{Mm}$.

Höhe des letzten Umganges . . . . . . 36 ,

Höhe des vorletzten Umganges . . . . . 18 ,

Grösste Dicke des letzten Umganges . . . . . 34 ,

Grösste Dicke des vorletzten Umganges . . . 18 ,

Bemerkungen. Unsere Art ist offenbar dem Ammonites subtricarinatus d'Orbigny, Prodrome II, p. 212 (Amm. tricarinatus d'Orbigny, Pal. Franç. I, p. 307, pl. 91, fig. 1, 2) nahe verwandt, indem sie mit diesem die geringe Wachsthumszunahme und Involubilität der Umgänge und die drei characteristischen Kiele des Bauches gemein hat. Die wesentliche Verschiedenheit beider heruht in der Art der Berippung und Knotenbildung. Denn andere Unterschiede wie das Convergiren der Seitenflächen gegen den Rücken und die stärkere Wölbung der Nabelfäche bei Amm. subtric. mögen in der verschiedenen Erhaltungsart beruhen. Bei Amm. subtric. liegen auf der Nabelkante etwa 20 Knoten, welche auf der Seitenfläche als Rippen fortsetzen, wobei die meisten dichotomiren, nur wenige einfach bleiben. Alle bilden am Rücken eine zweite Knotenreihe, so dass man hier 36 enge beisammen stehender Höcker zählt. Dieser eigenthümliche Schmuck der Seiten ist, soweit bis jetzt ermittelt, bei Ammonites subtric. durchaus constant. Denn d'Orbigny's Exemplaren von Sougraigne im Aude-Departement gleichen die Funde 
von den fernsten Lokalitäten vollkommen, wie die Exemplare aus Schlesien, welche Drescher auffand (Zeitschr. der deutsch. geol. Ges. Bd. XV, S. 331, Taf. VIII, Fig. 2-4), wie das westphälische Vorkommen (ebend. Jahrg. 1866, S. 72),- welches wir selbst aufheben, und wie die Erfunde von Stoliczka in Ostindien (Mem. of the Geol. Surv. of India, Palaeontologia indica III, 1, p. 54, tab. XXXI, fig. 3) darthun ${ }^{1}$.

Hiernach dürfte unsere Art als eine wohl verschiedene, gut begründete zu betrachten sein.

Torkommen. Ich erhielt das beschriebene Exemplar mit der Angabe, dass es bei Osterfeld (unweit Oberhausen) gefunden sei. Die Gesteinsbeschaffenheit, ein äusserst glaukonitreicher lockerer Mergel; widerspricht dieser Angabe nicht. Bei der überaus grossen Seltenheit von Ammoneen in diesem sonst fossilreichen Mergel mag jedoch darauf hingewiesen werden, dass zahlreiche Versteinerungen, in der Erhaltungsart nicht von denjenigen von Osterfeld zu unterscheiden, in einem auch Ammoneen umschliessenden Gesteine ebenfalls unweit Essen, bei Stoppenberg, gesammelt sind. Das Alter der Schichten von Stoppenberg ist noch nicht völlig genau ermittelt, aber muthmasslich ein wenig älter als dasjenige der Mergel von Osterfeld.

Geologishes Alter. Indem vorläufig Osterfeld als Fundort adoptirt wird, gehört unsere Art dem Senon an, welches durch Belemnitella quadrata charakterisirt wird und zwar dessen unteren Schichten.

Das Original befindet sich in der Sammlung des Herrn Sack in Essen.

\section{Erklärung der Abbildungen.}

Tafel V.

Fig. 1 a stellt das besehriebene Exemplar in natiirlicher Grösse von der Seite dar.

Fig. 1 b dasselbe Exemplar in horizontaler Lage gegen den Bauch und gegen die Mündung gesehen, wobei irrthiimlich die Mundhöhe etwas zu gross angegeben ist.

1 Dagegen scheint es gewagt, das mit einfachen, stark nach vorn geneigten und nur am Rücken mit einem Knoten versehenen Rippen verzierte Ammoniten-Bruchstück, welehes Gabb, Geol. Surv. of California Palaeont. tom. I, tab. X, fig. 4 darstellt, zn Ammonites subtricarinatus zu ziehen. Ebend. p. 60. 


\title{
Ammonites margae sp. $n$.
}

\author{
Taf. V, Fig. 2.
}

Schale gross, scheibenförmig, flach zusammengedrückt, mit weitem Nabel, auf dem Bauche gekielt, auf den Seiten mit Rippen und Knoten geziert. Die Umgänge nehmen etwas rascher an Höhe als an Breite zu, nur wenig, etwa $1 / 4$ involut. Man kennt nur die drei äussern Umgänge. Der innerste Theil der Schale bis zu $20 \mathrm{Mm}$. Durchmesser ist an keinem Stücke erhalten. Die Seiten flach gewölbt, gehen allmählig ohne Kante in den hoch gekielten Bauch über. Der Kiel reicht, wie man an den Abdrücken im Nebengestein bemerkt, noch scharf schneidig 4-6 $\mathrm{Mm}$. weiter vor als an den Kernen. Unsere Litbographie war schon vollendet, als wir dergleichen Stücke erhielten, und konnte also der Kiel in der Abbildung nur noch angedeutet werden.

Der innerste Umgang zeigt breite, kurze, wellenartige Rippen. Auf den folgenden Windungen sind die Rippen deutlicher, am ausgeprägtesten in der Gegend des Nabels, jenseits der halben Seitenhöhe werden sie undeutlicher, oft bis zum Verschwinden, und bilden dann in der Nähe des Bauches sehr breite rundliche Höcker, welche auf den inneren Windungen von den folgenden T'mgängen überdeckt werden.

Die Zahl der Rippen ist gering; sie wechselt zwischen 13 und 18 auf dem letzten Tmgange. An einem, dem abgebildeten Exemplare, stehen die letzten, der Mündung nahen Rippen gedrängter als gewöhnlich. Die Wohnkammer scheint wenigstens $2 / 3$ des letzten Umganges zu betragen. Die Nähte der Kammerwände sind nicht sehr deulich, doch erkennt man mit Bestimmtheit, dass sie nur wenig zerschnitten und verzweigt waren. Der erste Lateral-Lobus breit, gleich tief wie der Siphonal-Lobus, am Ende mit zwei Spitzen endigend ( $:)$; der zweite LateralLobus viel schmaler und weniger tief; endlich noch ein kleiner Auxiliar-Lobus.

Maasse (das grösste Exemplar misst $223 \mathrm{Mm}$.) :

\begin{tabular}{|c|c|c|}
\hline Durchmesser des Gehäuses . & & \\
\hline Höhe des letzten Umganges . & & \\
\hline$\eta \quad$ vorletzten Umganges & • & \\
\hline Nicht involuter Theil desselben & . & \\
\hline Dicke des letzten Umganges & & \\
\hline Höhe des Kieles & & \\
\hline
\end{tabular}


Geologische Verbreitung. Die Art bildet das wichtigste Cephalopod der sogenannten „grauen Mergel“, dem tiefsten Gliede der senonen Kreide im wcstphälischen Becken, und wurde namentlich im Schachte von der Heydt bei Herne beobachtet. Ein Exemplar, welches mir ebenfalls vorliegt, soll aus den Quadraten-Mergeln von Osterfeld stammen. Es gilt über dieses Vorkommen dasselbe, was über die Auffindung des Ammonites tridorsatus bei Osterfeld gesagt wurde.

Die der Beschreibung zu Grunde liegenden Originale befinden sich theils in der Sammlung der Bergschule zu Bochum, theils in der des Herrn Sack, theils in meiner Sammlung.

\title{
Erklärung der Abbildungen.
}

Tafel V.

Fig. 2 a Ein Exemplar in $\% / 3$ der natïrlichen Grösse von der Seite gesehen, 2 b dasselbe Exemplar in gleicher Verkleinerung gegen die Mündung und gegen den Bauch gesehen.

\section{Ammonites Westphalicus.}

\author{
Taf. VI, Fig. 2.
}

v. Strombeck, Zeitsahr. d. deutsch. geolog. Ges. Bd. XI, S. 56 .

Ausser Ammonites margae umschliesst der "graue Mergel" des südlichen Westphalens noch mehrere gekielte Ammoniten. Einer derselben ist bereits durch Herrn von Strombeck in mehreren Fxemplaren aufgefunden und an dem angeführten Ort, wie folgt, beschrieben worden. -Die Stücke haben bis zu 12 \%oll Durchmesser. Der Kiel ist ziemlich hoch und beiderseits mit einer Furche versehen. Mundöffnung mehr wio doppelt so hoch als breit. Seiten ganz flach mit radialen, abgerundeten und einfachen Rippen versehen, welche letztere an der Sutur entspringen und bis zur Rïckenkante fortsetzen, wo sie mit einem abgerundeten Hïcker endigen. Selten und ohne Regel schaltet sich in höherem Alter, von der Rückenkante ab bis zur Mitte der Höhe, eine schiefliegende, übrigens gleiche Rippe ein. Anzahl der Rippen bei 8 bis 12 Zoll Durchmesser $=25$ bis 30 pro T'mgang. An einem $3 / 4$ Zoll grossen Exemplare, das jedoch nicht gan\% entschieden derselben Species zugehört, zählt man 
nur 20 Rippen, die sich nächst der Sutur und am Rücken etwas stärker markiren als sonst. Involubilität sehr gering. Bei 10 Zoll Durehmesser hat der letzte Umgang 21/4 Zoll, der vorletzte $1^{1 / 2}$ Zoll Höhe, die Windungszunahme daher verhältnissmässig unbedeutend."

In einer kleinen Entblössung der "grauen Mergel“ zwischen Essen und Stoppenberg in der Nähe der Zeche Königin Elisabeth fand ich einen c. 9 Zoll im Durchmesser haltenden Ammoniten, welcher vielleicht der beschriebenen Art angehört. Das Stück ist gegenwärtig nur noch $6^{3 / 4}$ Zoll gross. Es zeigt die characteristische Bildung des Bauches und der Rippen, deren man am Nabel 19, an der Bauchkante 24 zählt. Breite und Höhe der Mündung verhalten sich wie 3:4. Ich gebe von dem Stücke nur die Bauchansicht Tafel VI, Fig. 2, indem die Seitenansicht und die Gestalt der Rippen und Knoten - abgesehen von der grösseren Zahl - mit derjenigen von Ammonites margae (Tafel V, Fig. 2a) übereinstimmt. Bei Vergleich dieser beiden Stïcke liegt die Frage nahe, ob es möglich sei, dass durch die Art des Versteinerungsprocesses unter Mitwirkung von Compression die den Kiel begleitenden Furchen, auf der ganzen Erstreckung, auf der äusseren und auf allen inneren Windungen durchaus verschwinden können, ohne dass zugleich eine Unregelmässigkeit in der Form des Bauches sich zeige? Wäre dies der Fall, so würde Ammonites Westphalicus in Bezug auf Bildung der Rippen sehr veränderlich sein. Aber ich habe das Stück von Essen zerbrochen und genau die inneren Windungen geprüft, an keiner Stelle jedoch wurde eine Spur von dem hohen characteristischen Kiele des Ammonites margae wahrgenommen, während derselbe, einmal beobachtet, auch bei den inneren Windungen aller Stücke dieser Art leicht zu finden ist. Ich glaube also nach dem mir vorliegenden Material beide Formen als verschiedene Arten betrachten zu müssen. Gehört das abgebildete Exemplar von Essen wirklich zu Amm. Westphalicus, so differirt die Art in Rücksicht auf die Zahl der Rippen und in Rücksicht auf das Verhältniss der Höhe und Breite stärker als Herr von Strombeck angibt. Ich besitze ein Bruchstück der Art von der Zeche Hannover unweit Bochum, also aus demselben Schachte, aus dem Herr von Strombeck einen grossen Theil seines Materiales erhielt. Dieses Bruchstück gehört einem Ammoniten von 6 Zoll Durchmesser an. Höhe und Breite wie 1:2, Zahl der Rippen c. 26, Bauch wie angegeben. Es liegt alsó 
hier wohl unzweifelhaft die durch Herrn v. Strombeck aufgestellte Art vor. Dieses Stück aber unterscheidet sich von Amm. margae sowohl, wie von dem besprochenen Exemplare von Essen noch durch die geringere Wachsthumszunahme, durch die grössere Weite der Spirale, welche auf vier bis fünf Umg:ïnge schliessen lässt.

Nach diesem Stücke zu urtheilen, steht Ammonites Westphalicus in Bezug auf Gestalt des Gehäuses den Ammonites tridorsatus nahe, die Rippen aber zeigen eine Bildung, welche derjenigen von Ammonites margae durchaus verwandt ist.

Geologische Verbreitung. Die Art gehört dem untersten Senon, den ,grauen Mergeln ${ }^{*}$ des südlichen Westphalens an und wurde namentlich beobachtet im Schachte Carl der Zeche Hannover bei Gelsenkirchen, ferner auf der Grube Shamrock bei Herne, auf dem Schachte Carl bei Altenessen und der Königsgrube bei Gelsenkirchen.

\title{
Erklärung der Abbildungen. \\ Tafel VI.
}

Fig. 2 stellt das beschriebene, aus der Nähe von Essen herrührende Exemplar gegen den Bauch gesehen in natürlicher Grösse dar.

\section{Ammonites Texanus.}

\author{
Taf. VI, Fig. 1. 3.
}

1×49. Ammonites Texanus. Römer, Texas. Mit besonderer Rücksicht auf deutsche Auswanderung etc. S. 417 .

1852. Ammonites Texanus. Römer, die Kreidebildungen von Texas und ihre organischen Einschliusse, S.31, Taf. III, Fig. 1 a-e.

18.78. Ammonites Texanus. F. von Hauer, Ueber die Cephalopoden der Gosauschichten, in Hawer: Beiträge z. Paläontologie I, 1. S. 10, Taf. II, Fig.4-6.

Die Bestimmung des einzigen, in ziemlich vollständiger Erhaltung vorliegenden Exemplares ist nicht ganz zweifellos. Indem ich auf die Peschreibung der Art bei Römer und von Hauer verweise, soll hier nur das Verhältniss angedcutet werden, in wclchem unsere Form zu dem Texanischen Vorkommen und dem der Gosau steht. Der Gesammthabitus stimmt bei allen überein. Die grössere Höhe und geringere Dicke 
der Windungen an unserm Stücke ist der erlittenen Compression zuzuschreiben. Die Exemplare des Gosauthales sind alle, wie von Hauer ausdrücklich angibt, etwas verdrückt, deshalb stimmt auch unser Exemplar besser mit der Abbildung von v. Hauer, als mit der von Römer gegebenen. Die Zahl der Windungen hat nur v. Hauer mit Sicherheit observiren können. Die Schale bestand aus etwa sechs, langsam an Höhe und Breite zunehmenden, sich beinahe nur berührenden Umgängen (v. H.). Weder an unserem, noch an den vorliegenden Originalen aus Texas sind die innersten Windungen sichtbar oder erhalten.

Die Zahl der starken, radialen Rippen beträgt bei dem grössten Texanischen Stücke nur 22, an unserem nur wenige Linien kleineren Exemplare 26 auf dem äusseren Umgange, v. Hauer zählte an seinem Material sogar bis zu 31 Rippen. Offenbar variirt die Zahl der Rippen bei Ammonites Texanus, denn es liegt mir noch ein von Römer selbst bei Neu-Braunfels in Texas aufgelesenes Bruchstück derselben Art vor, welches bei gleicher Grösse mit dem von Römer abgebildeten grössten Exemplare, viel gedrängter stehende Rippen als letzteres zeigt und in der Zahl derselben genau mit derjenigen unseres Stückes übereinstimmt. Der Umstand, dass das Westphälische Vorkommen breitere und flachere Rippen zeigt, als das Texanische, dürfte wiederum dem erlittenen Drucke zuzuschreiben sein. Wichtiger ist, dass bei fortgeschrittenem Wachsthum die Rippen unseres Stückes eine grosse Neigung nach rückwärts erhalten, aber auch diese Differenz ist scheinbar, indem sie durch das GosauVorkommen vermittelt wird. Die Bildung der die Rippen verzierenden Knoten ist überall dieselbe. Die inneren Knoten sind rund und stumpf, die zwei äusseren spiralen Reihen derselben dagegen in die Länge gezogen, besonders die der äussersten Reihe, schon dem Bauche angehörend, sind sehr verlängert, und zahnartig vorspringend überragen sie selbst den glatten Kiel des Bauches. Bei den fremden Vorkommnissen trägt jede Rippe fünf Knoten, unser Exemplar nur vier. Auf dieses verschiedene Verhalten ist weniger Gewicht zu legen, als es auf den ersten Blick scheinen könnte. Auch die grossen Originalexemplare von Texas zeigen nur die dem Bauche zunächst stehenden Knotenreihen vollkommen deutlich; auch die dem Nabel zunächst stehende Reihe ist noch leidlich erkennbar, viel weniger die dritte, die zweite aber kaum sichtbar, oder gar nicht vorhanden. Unter diesen Umständen dürfte auch 
auf das Fehlen der zweiten Knotenreihe an unserem Exemplare kein Gewicht zu legen sein, um so weniger, als Form und Lage der vorhandenen Knotenreihen mit dern Texas- und Gosau-Vorkommen ganz übereinstimmen. Es stehen namentlich die äusseren Reihen näher zusammen, als die inneren. Auch das letzte Bedenken wird schwinden, wenn wir das Tafel VI unter Figur 3 abgebildete Bruchstück, welches ich in den gelblichen, glaukonitischen Mergeln bei Stoppenberg auffand, mit in Betracht ziehen. An diesem Stücke ist die zweiten Knotenreihe vorhanden, aber es fehlt die erste, dem Nabel zunächststehende. Von diesem Mangel abgesehen, stimmt dieses Bruchstück überhaupt sehr gut mit den Exemplaren aus Texas, namentlich in Bezug auf den Umriss der Mündung und die Form der Rippen, da es kaum merklich verdrückt ist. Demnach dürfte es, trotz des geringen mangelhaften Materials als erwiesen gelten, dass Ammonites Texanus auch der norddeutschen Kreide angehöre.

Es muss noch der Beziehungen gedacht werden, in welchen unsere Art zu anderen Ammoniten steht. Römer fand eine Verwandtschaft mit Rhotomagensis, v. Hauer mit einzelnen Varietäten von Ammonites Coupei und führt namentlich dafür die Abbildung bei Sharpe Taf. XIX Fig. 1 an. Grösser ist die Aehnlichkeit unseres Stückes mit Amm. rostratus Sow. Taf. 173, welcher ziemlich allgemein für eine Varietät des Ammonites inflatus gilt. Am nächsten ist unsere Art den Cristaten verwandt, welche Baily aus der. Kreide Süd-Africas beschrieb, nämlich dem Amm. Stangeri und Amm. Soutanii (Quat. Journ. Geol. Soc. London, Vol. XI, p. 455, tab. XI, fig. 1, fig. 2), zwischen welchen beiden Ammonites Texanus in der Mitte steht. Auch Ammonites Bourgeoisianus d'Orb. (Prod. II, p. 112, No 16) dürfte verwandt sein, so weit nach der mangelhaften, von keiner Abbildung begleiteten Beschreibung zu urtheilen ist.

In gewissem Alter erinnert auch der veränderliche Ammonites Woolgari (vergl. Sharpe, tab. XI) an unsere Art, namentlich wenn bei dieser einzelne Knotenreiben undeutlich werden. Amm. Woolgari trägt deren nie mehr als 3 auf einer Rippe. Sicher unterscheidend auch bei kleineren Bruchstücken ist der unterbrochene, sägenartige Kiel. Zudem dürfte Amm. Woolgari ein etwas höheres Alter haben. Ich habe ihn bisher nur im Brongniarti-Pläner beobachtet. 
Geologisches Vorkommen. Die beiden beschriebenen Exemplare gehören dem untersten Senon des Westphälischen Beckens an. Das unter Fig. 1 abgebildete Exemplar, welches ich dem Herrn Bergreferendarius Volmer verdanke, stammt aus den grauen Mergeln des Schachtes von der Heydt bei Herne, das unter Fig. 3 abgebildete Fragment fand ich in den gelblichen, glaukonitischen Mergeln bei Stoppenberg.

\section{Erklärung der Abbildungen. Tafel VI.}

Fig. 1 a stellt ein von der Seite, $1 \mathrm{~b}$ dasselbe gegen den Bauch und die Mündung gesehene Exemplar, aus den grauen Mergeln von Herne stammend, dar.

Fig. 3 Bruchstück einer Windung von der Seite gesehen, von Stoppenberg bei Essen.

\section{Ammonites Hernensis n. sp.}

Taf. VI, Fig. 4.

Das Gehäuse besteht aus drei ungefähr zur Hälfte involuten Umgängen, deren gerundeter Bauch sich ohne Kante mit den Seiten verbindet. Die Oberfläche des Steinkernes ist mit zahlreichen flach gerundeten Rippen bedeckt, die am Bauche eine Biegung nach vorn machen. Neben den Rippen finden sich diesen parallel und gleichfalls am Bauche eine Bucht nach vorne bildend, sechs Einschnürungen auf dem äusseren Umgange. Weder die Rippen noch die Einschnürungen erreichen den Nabel. Die letzteren werden in der Nähe der Nabelkante von einem runden oder etwas verlängerten Höcker begränzt. Die Grösse des Stückes beträgt $66 \mathrm{Mm}$.

Nach Gestalt, wie nach Oberflächenverzierung steht unsere Art dem Ammonites planulatus Sow. ${ }^{1}$ so nahe, dass sie eben nur durch die Höckerreihe davon unterschieden ist. Denn die Zahl der Einschnürungen ist schwankend. Ursprünglich wurden der Art nur 4 oder 5 Furchen zugeschrieben. Seitdem aber Ewald ${ }^{2}$ nachgewiesen hat, dass auch

1 Tab. $570=$ Amm. majorianus d'Orb. tab. 79 .

2 Zeitschr. d. deutsch. geol. Ges. II, 445 . 
Ammonites Emerici Raspail ${ }^{1}$ mit Ammonites majorianus d'Orb. zu vereinen sei, steht es fest, dass die über Seiten und Bauch eines Umganges laufenden Furchen zwischen vier und sechs wechseln. Ebensowenig liegt in der Rippenbildung zwischen der alten und neuen Art eine Verschiedenheit, da die Behauptung, welche freilich auch von Ewald von Neuem aufgestellt ist, dass die Rippen nur an der Oberfläche der Schale, nicht aber am Kerne sichtbar seien, sich nicht bestätigt hat, wie ich bereits früher nachwies ${ }^{2}$. Auch Pictet und F. von Hauer haben dieselbe Beobachtung gemacht ${ }^{3}$.

Die genannten Höcker dagegen, welche unsere Art in der angegebenen Weise trägt, habe ich bei keinem der zahlreichen Exemplare des Amm. planulatus aus Gault oder Cenoman, welche ich zu untersuchen. Gelegenheit hatte, beobachtet, und ebenso wenig sind sie in den zahlreichen Darstellungen, welche wir von dieser Art durch Sowerby, d'Orbigny, Pictet und Sharpe besitzen, angegeben worden, so dass ich glaube, darin cinen Grund zur spccifischen Trennung finden zu müssen. Hierzu gesellt sich noch der Umstand, dass ein echtcr unzweifclhafter Amm. planulatus, trotz der grossen horizontalen und vertikalen Verbreitung, bisher noch niemals in so jungen Schichten beobachtet wurde.

Gcologisehes Vorkommen. Das beschriebene und abgebildete Exemplar wurde von dem Herrn Bergreferendarius Volmer in den untersenonen „grauen Mergeln" des Schachtes von der Heydt bei Herne in Westphalen aufgefunden.

\section{Erklärung der Abbildungen.}

Tafel VI.

Fig. 4 stcllt das beschriebene Exemplar in der Seitenansicht dar. fig. 1-3.

1 Ann. des Scienc. d'observ. III, tab. 12, fig. 6. d'Orb. Terr. eret. tab. 51,

2 Zeitschr. d. deutsch. geol. Ges. Bd. XVIII, p. 72.

3 Sitzungsberichte d. kais. Ak. d. Wiss. Bd. XLIV, p. 655. 
Taf 1
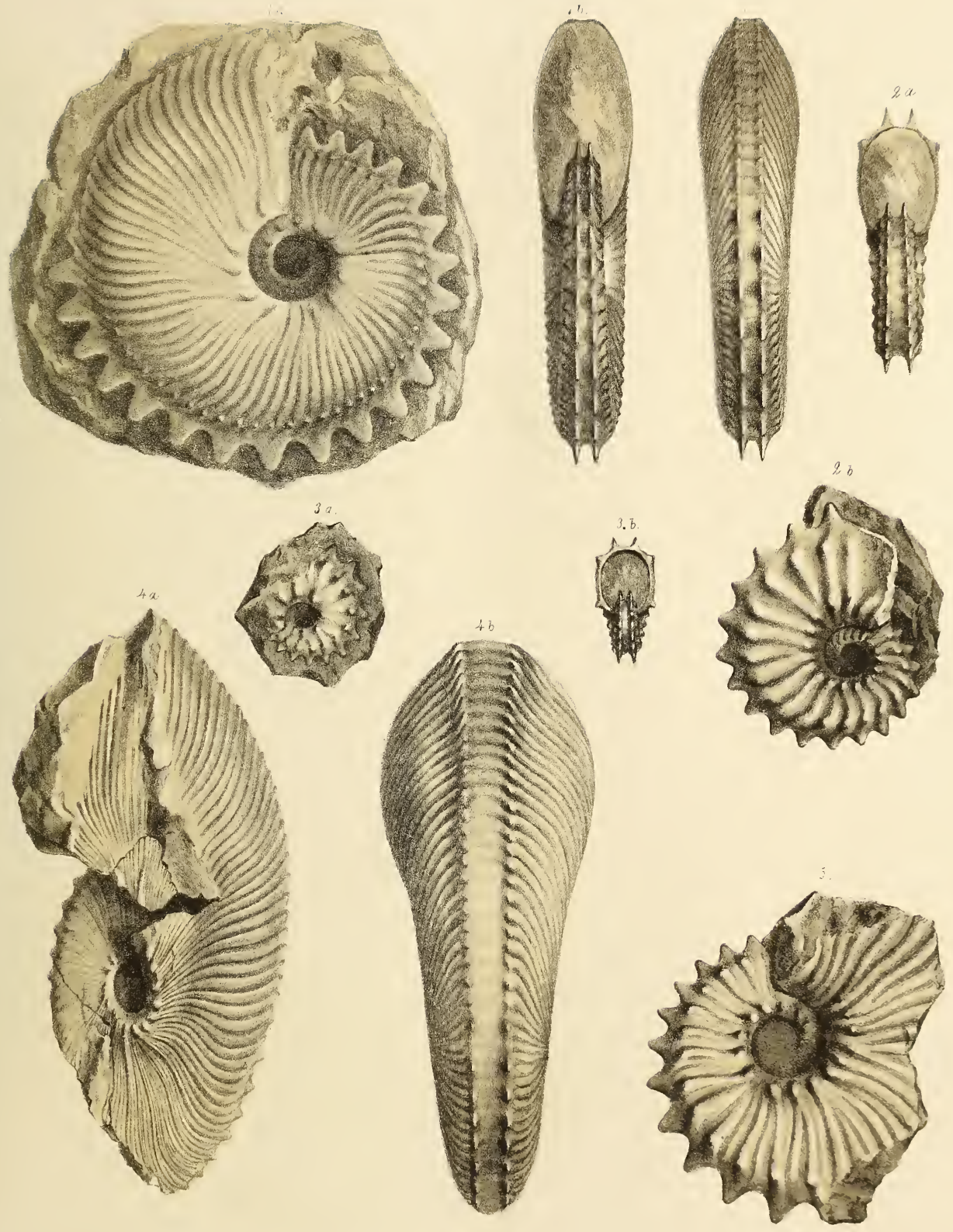


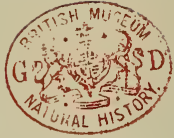





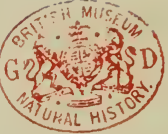


'Hattlii
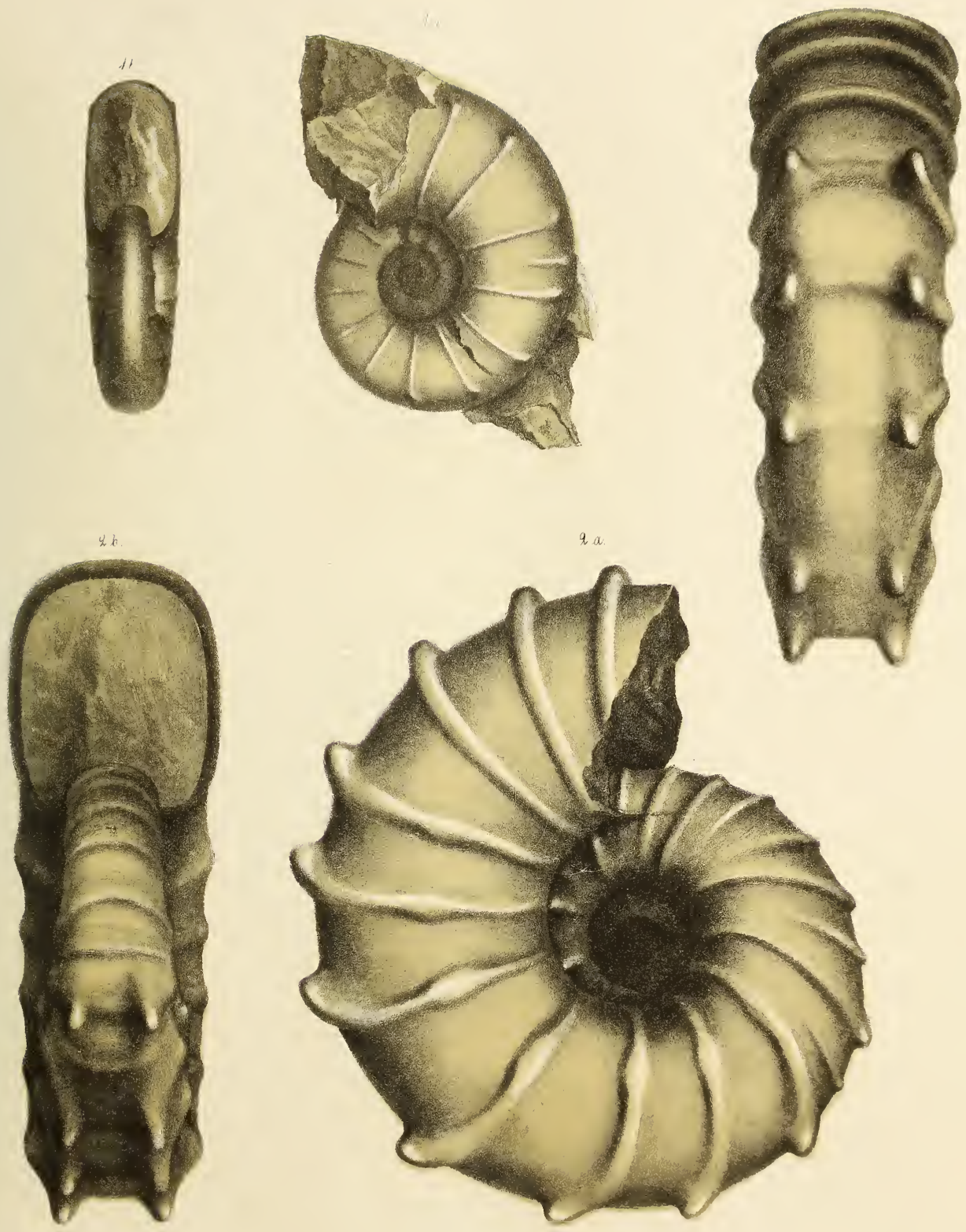


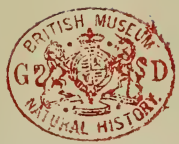




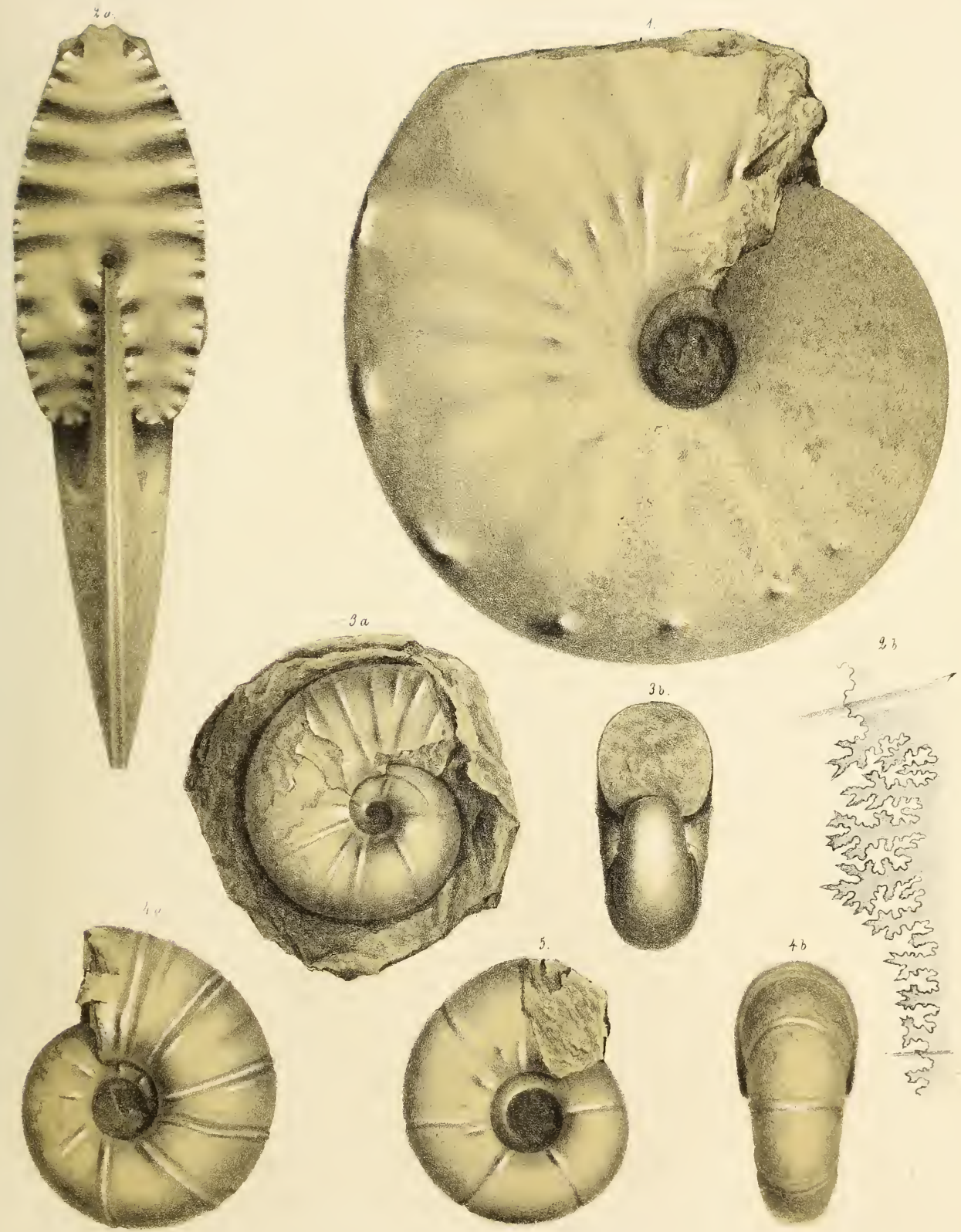





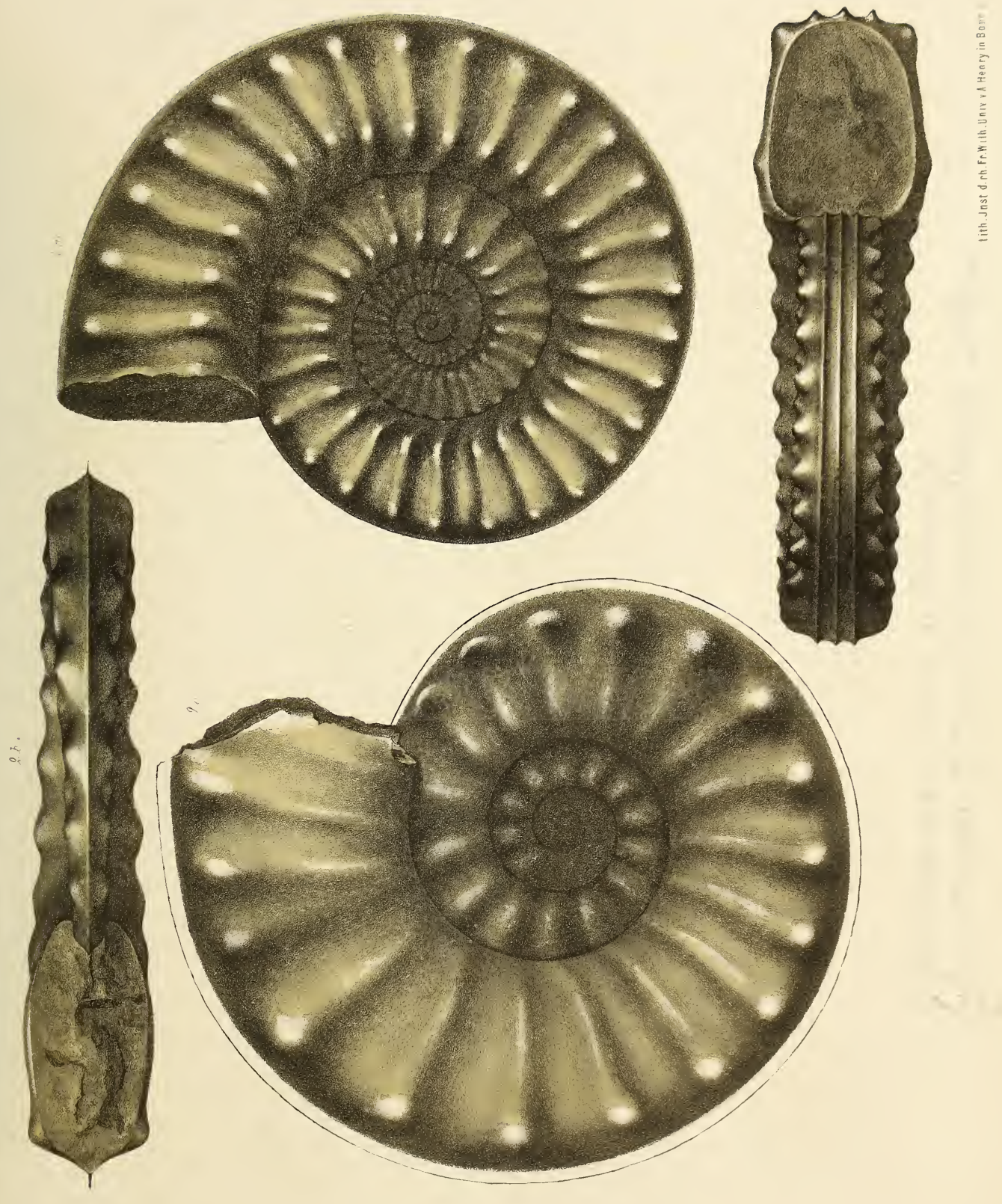



'l'a f.VI
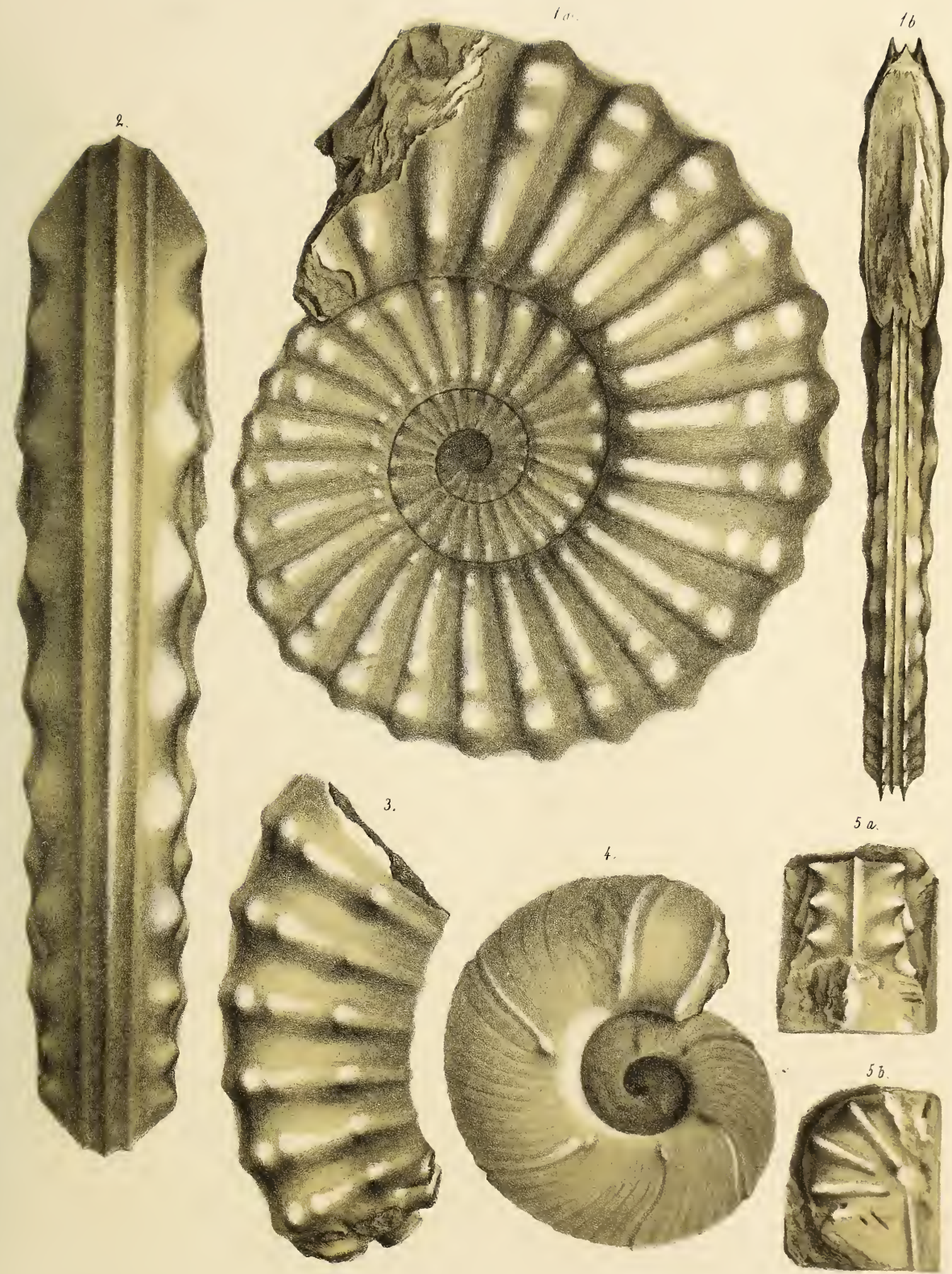
















































